\title{
Clinical characteristics and laboratory biomarkers changes in COVID-19 patients requiring or not intensive or sub-intensive care: a comparative study
}

Anna Maria Cattelan ${ }^{1 *}$ D, Eugenia Di Meco ${ }^{1}$, Marco Trevenzoli ${ }^{1}$, Alessia Frater ${ }^{1}$, Anna Ferrari ${ }^{1}$, Marco Villano ${ }^{2}$, Federica Gomiero², Giovanni Carretta ${ }^{3}$ and Lolita Sasset ${ }^{1}$

\begin{abstract}
Background: Identifying risk factors for severe novel-coronavirus disease (COVID-19) is useful to ascertain which patients may benefit from advanced supportive care. The study offers a description of COVID-19 patients, admitted to a general ward for a non-critical clinical picture, with the aim to analyse the differences between those transferred to the intensive (ICU) and/or sub-intensive care (SICU) units and those who were not.

Methods: This observational retrospective study includes all COVID-19 patients admitted to the Infectious Diseases Unit. Clinical, laboratory, radiological and treatment data were collected. The primary outcome was a composite of need of transfer to the ICU and/or SICU during the hospitalization. Patients who did not require to be transferred are defined as Group 1; patients who were transferred to the ICU and/or SICU are defined as Group 2.

Demographic, clinical characteristics and laboratory findings at the 1st, 3rd and last measurements were compared between the two groups.

Results: 303 were included. The median age was 62 years. 69 patients (22.8\%) met the primary outcome and were defined as Group 2. The overall fatality rate was $6.8 \%$. Group 2 patients were predominantly male $(76.8 \%$ vs. $55.1 \%$, $p<0.01)$, had a higher fatality rate $(14.5 \%$ vs. $3.8 \%, p<0,01)$, had more hypertension $(72.4 \%$ vs. $44 \%, p<0,01)$ and diabetes $(31.9 \%$ vs. $21 \%, p=0.04)$ and were more likely to present dry cough $(49.3 \%$ vs. $25.2 \%, p<0.01)$. Overall, chest X-ray at admission showed findings suggestive of pneumonia in $63.2 \%$, and Group 2 were more likely to develop pathological findings during the hospitalization (72.7\% vs. 17.2\%, $p=0.01$ ). At admission, Group 2 presented significantly higher neutrophil count, aspartate-transaminase and C-Reactive-Protein. At the 3rd measurement, Group 2 presented persistently higher neutrophil count, hepatic inflammation markers and CReactive-Protein. Group 1 presented a shorter duration from admission to negativization of follow-up swabs (20 vs. 35 days, $p<0.01)$.

Conclusions: The presence of comorbidities and the persistent observation of abnormal laboratory findings should be regarded as predisposing factors for clinical worsening.
\end{abstract}

Keywords: COVID-19, SARS-CoV-2, Novel coronavirus, Disease severity, Outcome, Intensive care, Sub-intensive care

\footnotetext{
* Correspondence: annamaria.cattelan@aopd.veneto.it

'Infectious Diseases Unit, Azienda Ospedale Università di Padova, Via Nicolò Giustiniani 2, 35128 Padova, Italy

Full list of author information is available at the end of the article
} 


\section{Background}

The outbreak of severe acute respiratory syndrome coronavirus-2 (SARS-CoV-2) causing the human disease named coronavirus disease (COVID-19) was first reported in China in December 2019 and declared a pandemic by the World Health Organization in March 2020 [1]. The first known cases of local transmission were detected in Italy at the end of February 2020. The northern regions of the country initially have been the most affected by the outbreak [2]. To address the emergency, strict social containment measures have been adopted and health care systems have been reorganized to cope with the enormous increase in the numbers of acutely ill patients [3, 4].

Hospital admission rates for patients with COVID-19 may vary substantially between countries, because of the different prevalence in infection and community testing rates and non-homogeneous admission criteria. However, it is estimated that 10 to $20 \%$ of adults presents clinical conditions requiring hospitalization, and in the majority of the cases this is due to respiratory distress [5]. The decision about location of care and clinical management depends on various factors, including clinical presentation, disease severity, need for supportive care, presence of risk factors for severe Diseases, and conditions at home. While mild to moderate disease in low-risk patients can be managed at home or in primary or secondary level healthcare facilities, severe and critical diseases need tertiary hospitals where high dependency/ sub-intensive (SICU) or intensive care units (ICU) are available [6]. Because the provision of intensive care and the availability of resources is limited [7], the identification of risk factors for severe infection is crucial for clinicians to identify patients who may benefit from aggressive supportive care and for health and government officials to adequately address local outbreaks.

The percentage of patients requiring ICU has ranged from 5 to $37 \%$ [8-13]. The most common reason for intensive care unit admission is hypoxemic respiratory failure leading to mechanical ventilation (MV) or noninvasive ventilation (NIV) [14]. In a large cohort study performed on ICU admitted patients in northern Italy $88 \%$ of the patients required MV and $11 \%$ NIV [15]. Patients admitted to intensive care units are reported to be older and predominantly male, and to have more frequently existing comorbidities such as hypertension, heart failure, renal disease, and obesity. Lower lymphocyte count, elevated serum troponin, C-Reactive Protein, D-dimer, and white blood cell count are also more commonly seen in patients presenting with severe infections $[10,12,13,16,17]$.

The aim of our study is to describe the clinical characteristics and the dynamic changes of laboratory parameters of the patients admitted to the Infectious Diseases
Unit (IDU) of the University Hospital of Padua. In order to identify possible predictors of clinical worsening we compared the results between the group of patients who required being transferred to the ICU and/or SICU during the course of the hospitalization and the patients who did not.

\section{Methods}

\section{Study design}

This is a retrospective observational study of prospectively collected data of adult patients hospitalized at the IDU of the University Hospital of Padua, Veneto region, Italy. The IDU is one of the general wards where COVID-19 patients are admitted with a clinical picture not requiring intensive support. We included all adult patients (aged $\geq 18$ years), admitted between February 22 and May 20, 2020, with laboratory-confirmed SARSCoV-2 infection and hospitalized for $\geq 24 \mathrm{~h}$. In order to include only the patients initially hospitalized with a non-critical clinical picture, in the analysis the patients who required being transferred to the ICU/SICU within $12 \mathrm{~h}$ from admission and those that were transferred to the IDU after a permanence in the ICU/SICU are excluded.

We defined the primary outcome as a composite endpoint of need of transfer to the ICU and/or the SICU during the hospitalization. The group of patients who did not require to be transferred are defined Group 1; the patients who were transferred to the ICU and/or SICU are defined Group 2. Criterion for the transfer to the SICU was the need for NIV (defined as assisted ventilation that delivers positive pressure throughout the respiratory cycle with additional phasic increases in airway pressure, without the presence of an endotracheal tube [18]). Criteria for the transfer to the ICU were the need for MV or NIV and/or the occurrence of shock or organ failure.

Local ethics committees were notified about the study protocol. The study was performed according to the ethical guidelines of the Declaration of Helsinki (7th revision).

\section{Data collected}

Demographic, clinical, laboratory and treatment data were extracted from paper and electronic medical records using a standardised data collection form.

Laboratory confirmation of SARS-CoV-2 infection was obtained by the detection in respiratory specimens (throat-swab) by an in-house real-time RT-PCR method according to Lavezzo et al. [19]. Throat-swabs were performed by using flocked swabs in liquid-based collection and transport systems (eSwab ${ }^{\odot}$, Copan Italia Spa, Brescia, Italy). Follow up swabs were performed every 3 to 5 days. A patient was considered negative for SARS-CoV-2 when two throat-swabs, performed in consecutive days, 
resulted negative. In the case patients were still positive at discharge, the repetition of follow-up swabs was performed through the territorial health services from which data were collected.

Laboratory tests included: blood count, CD4 T lymphocyte count, activated partial thromboplastin time (APTT), prothrombin time (PT), alanine aminotransferase (ALT), aspartate aminotransferase (AST), gammaglutamyl-transferase (GGT), alkaline phosphatase (ALP), total bilirubin, urea, creatinin, glomerular filtration rate calculation (GFR), C-Reactive Protein (CRP), procalcitonin and D-Dimer. The treating physician, according to patients' clinical needs, decided the frequency of repetition of laboratory tests.

In order to describe the dynamic changes of laboratory tests and possible early patterns indicating a deterioration of clinical conditions, the 1st measurement available (at admission), the 3rd measurement since admission, and the last measurement (before discharge or death) were analysed. The choice to consider the 3rd measurement was dictated by two main factors: 1 ) the 3rd measurement was always performed between the 3rd and the 5th day of hospitalization; 2) the median time from hospital admission to ICU/SICU transfer was of 5 days.

Laboratory tests to exclude the presence of bacterial infections (blood and urine cultures, pneumococcal and legionella urinary antigen tests, Chlamydia pneumoniae and Mycoplasma pneumoniae serology) were also performed at admission and during the hospitalization in case of a clinical suspicion of superinfections.

Chest radiographs were done for all patients at admission and repeated according to clinical needs. They were considered positive in case of evidence of singular or multiple consolidations and/or interstitial opacities.

Anti SARS-CoV-2 drugs administered during the hospitalization for $>2$ days were recorded. Criteria for the choice of the therapeutic regimens for COVID-19 followed national guidelines [20] and physicians' judgements. As a general rule, patients with comorbidities, age $>70$ years, respiratory symptoms and/or evidence of pneumonia were treated with chloroquine (CQ) $(500 \mathrm{mg}$ orally twice daily) or hydroxychloroquine (HCQ) (200 mg orally twice daily). CQ and HCQ could be associated with lopinavir/ritonavir ( $400 \mathrm{mg} / 100 \mathrm{mg}$ twice daily) or azithromycin (500 mg orally daily); standard duration of therapies was 5-7 days. In addition, tocilizumab and remdesivir were also used. Intravenous high dose glucocorticoids were used only in severe or critical patients. All patients, if not contraindicated, received thromboprophylaxis with daily low-molecular weight heparin.

\section{Statistical analysis}

Continuous and categorical variables were presented as median (IQR) and $n$ (\%), respectively. We used the $R$ software (version 3.6.2) to perform Mann-Whitney U test, $\chi^{2}$ test, or Fisher's exact test to compare differences between Group 1 and Group 2 patients where appropriate. A $p$-value less than 0.05 was considered statistically significant.

\section{Results}

A total of 325 adult patients were hospitalised with a confirmed diagnosis of COVID-19 in the study period. Twenty-two patients were excluded because they met the exclusion criterion of being admitted to the ICU or SICU within $12 \mathrm{~h}$ since admission or before to the permanence in the IDU. Therefore, a total of 303 patients were included in the analysis.

The median age was 62 years (IQR 50-74) and 182 (60.1\%) were men. The fatality rate was $6.8 \%$. All deaths were due to respiratory failure. The overall median duration of hospitalization was 9 days (IQR 6-16). 69 patients $(22.8 \%)$ met the composite outcome. Of those, 15 were transferred to the ICU, 36 to the SICU and 18 to both of the units. The median time from hospital admission to the transfer was 5 days (IQR 2-13.5).

Table 1 summarizes the main demographics characteristics of the sample and the differences between the two groups of patients: group 2 patients were predominantly male $(76.8 \%$ vs. $55.1 \%, p<0.01)$, had a significantly higher fatality rate $(14.5 \%$ vs. $3.8 \%, p<0.01)$ and a longer hospitalization ( 18 vs. 7 days, $p<0.01$ ). The difference between the median age of the two groups did not reach statistical significance $(p=0.06)$.

From the 284 discharged patients, 185 (65.1\%) went home, while $7(2.5 \%), 32(11.3 \%)$ and $17(5.9 \%)$ were transferred to a nursing home/community healthcare facility, a secondary level hospital or to a rehabilitation institute respectively. Group 2 patients were less likely to be discharged at home ( 47.5 vs. $69.8 \%, p=0.02)$ and were more often transferred to a secondary level hospital $(18.6 \%$ vs. $9.3 \%, p=0.03)$ or to a rehabilitation institute ( $15.2 \%$ vs. $3.5 \%, p=0.80$ ) compared to Group 1 .

Table 2 shows the clinical presentation at admission and the coexisting medical conditions. Fever (80.2\%), dyspnoea (31.7\%) and dry cough (30.7\%) were the most common symptoms. Fever, which generally is considered being the most important symptom (as confirmed in this study), reached no statistically significant difference between the two groups $(p=0.73)$, while a significant difference was detected for the presence of dry cough (25.2\% vs. $49.3 \%, p<0.01)$.

Hypertension $(72.4 \%$ vs. $44 \%, p<0.01)$ and known or newly diagnosed diabetes ( $31.9 \%$ vs. $21 \%, p=0.04)$ were more common among patients in Group 2 compared to Group 1. Patients with no comorbidities were less likely to belong to Group $2(20.3 \%$ vs. $37.2 \%, p=0.03)$. 
Table 1 Demographic characteristics and outcomes of non-ICU/SICU (Group 1) and ICU/SICU (Group 2) patients

\begin{tabular}{|c|c|c|c|c|c|}
\hline & Available & $\begin{array}{l}\text { All patients } \\
\text { n.303 }\end{array}$ & $\begin{array}{c}\text { Group } 1 \\
\text { n.234 (77.2\%) }\end{array}$ & $\begin{array}{c}\text { Group 2 } \\
\text { n.69 (22.8\%) }\end{array}$ & $p$ \\
\hline Sex male & $100 \%$ & $182(60.1 \%)$ & $129(55.1 \%)$ & $53(76.8 \%)$ & $<0.01$ \\
\hline Age, years & $100 \%$ & $62(50-74)$ & $60(47-72)$ & $68(56-77)$ & 0.06 \\
\hline Death & $100 \%$ & $19(6.8 \%)$ & $9(3.8 \%)$ & $10(14.5 \%)$ & $<0.01$ \\
\hline Length of hospitalization, days & $100 \%$ & $9(6-16)$ & $7(5-12)$ & $18(14-26)$ & $<0.01$ \\
\hline Patients discharged & $100 \%$ & $284(93.7 \%)$ & $225(96.1 \%)$ & 59 (85.5\%) & \\
\hline \multicolumn{2}{|l|}{ - Discharged at home } & $185(65.1 \%)$ & $157(69.8 \%)$ & $28(47.5 \%)$ & 0.02 \\
\hline \multicolumn{2}{|c|}{ - Transferred to a nursing home, community health facility, hospice } & $7(2.5 \%)$ & $5(2.2 \%)$ & $2(3.4 \%)$ & 0.70 \\
\hline \multicolumn{2}{|l|}{ - Transferred to a secondary level hospital } & $32(11.3 \%)$ & $21(9.3 \%)$ & $11(18.6 \%)$ & 0.03 \\
\hline \multicolumn{2}{|l|}{ - Transferred to a rehabilitation facility } & $17(5.9 \%)$ & $8(3.5 \%)$ & $9(15.2 \%)$ & 0.80 \\
\hline \multicolumn{2}{|l|}{ - Data not available } & $43(15.1 \%)$ & $34(15.1 \%)$ & $9(15.2 \%)$ & \\
\hline
\end{tabular}

Data presented as median (IQR) or as percentage. In the second column data availability is also shown

ICU Intensive Care Unit, SICU Sub-intensive Care Unit

Chest X-ray at admission to the IDU showed pulmonary lesions in $63.2 \%$ of the patients. No significant statistical differences were found between the two groups. Conversely, Group 2 patients were more likely to develop de novo pathological findings at the chest $\mathrm{X}$-ray during the course of hospitalization $(72.7 \%$ vs. $17.2 \%, p=0.01)$.

Laboratory findings at the 1st, 3rd and last measurements since admission are shown in Table 3 (see Additional file 1). Group 2 patients presented at admission a significantly higher neutrophil count, higher AST and CRP levels. At the 3rd measurement since admission, significant differences were found for white blood cell and neutrophil count, hepatic inflammation markers (AST, ALT, total bilirubin), and CRP. The GFR and the APTT resulted also significantly different between the two groups, but their median values were, in both cases, within the normal ranges. At discharge, Group 2 patients were found to have a significantly lower level of haemoglobin.

None of the patients resulted positive to the laboratory tests for bacterial infections (data not shown).

Table 2 shows data regarding antiviral drugs use. CQ or $\mathrm{HCQ}$, lopinavir/ritonavir and azithromycin were the most common drugs, prescribed during the course of hospitalization to $60.3,43.3$ and $39.6 \%$ of the patients respectively. Group 2 patients were less likely to receive CQ or HCQ ( $49.2 \%$ vs. $63.7 \%, p<0.01)$, azithromycin ( $30.4 \%$ vs. $42.3 \%, p=$ 0.01 ) and remdesivir (1.4\% vs. $7.7 \%, p=0.01$ ), and more likely to receive lopinavir/ritonavir ( $36.2 \%$ vs. $26.9 \%, p=0.01$ ).

Among Group 2 patients, $88.4 \%$ required NIV and 43.4\% MV.

As of June 1, 2020, 83.1\% of patient resulted negative for SARS-CoV-2 at the follow-up swabs. The median duration from hospital admission to negativization (considering the date of the second swab) was 22 days. Patients of Group 1 presented a significant shorter duration to negativization compared to Group 2 (20 vs. 35 days, $p<0.01)($ Table 1$)$.

\section{Discussion}

In this observational comparative study, clinical characteristics and laboratory biomarkers of a cohort of 303 patients with a confirmed SARS-CoV-2 infection and primarily hospitalized in the general ward of the Infectious Diseases Unit of Padua were analysed. 22.8\% of the patients (Group 2) required to be transferred to the ICU and/or SICU and overall 19 patients (6.8\%) died.

Our sample included only patients who presented at admission a non-critical clinical picture and did not primarily required intensive support. This could explain why our fatality rate was lower than those reported in recent European and American inpatient cohorts, ranging from 24 to $33 \%$ [11-13]. Moreover, population and patients' characteristics and prevalence of community testing can also partly explain the differences. The admission rate to the intensive/sub-intensive care observed in our cohort is relatively high when compared with previous studies (rates comprised between 5 and 37\%) [8-13]. It can be argued that a more extended aggressive support, especially in the form of a timely ventilator assistance, may guarantee a better management with higher chance of recovery of unstable or border-line patients.

Group 2 patients had a longer hospital stay and required more frequently to be transferred to a secondary level healthcare facility or to a rehabilitation institute compared to Group 1. These results may provide health officials and policymakers a better understanding on the interventions needed to properly address also long term consequences of local outbreaks.

Consistently with other studies [8-17], men were more represented than women and were at higher risk for severe disease. It has been reported that the reduced predisposition of females to viral infections could be attributed to the protection given by the $\mathrm{X}$ chromosome and sex hormones, which play an important role in the innate and adaptive immunity [21]. 
Table 2 Clinical, radiological characteristics, treatments and follow-up swabs of Group 1 and Group 2 COVID-19 patients

Co-existing medical conditions
Hypertension
Diabetes mellitus (pre-existing and newly diagnosed)
Chronic cardiac disease
Chronic pulmonary disease
Chronic gastrointestinal disease
Active malignancy
Transplant
Urologic disorders
Chronic kidney disease
Obesity (BMl > 30)
Overweight (BMl > 25)

Number of medical conditions

0

$\geq 2$

Symptoms at admission

Fever
Dry cough
Productive cough
Sore throat
Dyspnoea
Conjunctivitis
Diarrhoea
Myalgia
Arthralgia
Malaise
Dysgeusia
Skin rash

Sat02 $<94 \%$ in air room at admission

Positive chest $\mathrm{X}$-ray at admission

Positivization of chest X-ray during hospitalization

Anti SARS-CoV-2 treatment

Chloroquine or Hydroxychloroquine

Lopinavir/ritonavir

Remdesivir

Tocilizumab

Azithromycin

\section{Antibiotic treatment iv}

\section{0xygen therapy}

Low or high flow systems ${ }^{a}$

NIV

MV

\section{Available}

$100 \%$

All patients

n. 303

$153(50.5 \%)$
$71(23, .4 \%)$
$44(14,5 \%)$
$26(8.6 \%)$
$26(8.6 \%)$
$26(8.6 \%)$
$3(1.0 \%)$
$36(11.9 \%)$
$15(4.9 \%)$
$51(16.8 \%)$
$154(50.8 \%)$

$100 \%$

$101(33.4 \%)$

$96(31.7 \%)$

$100 \%$

(1)

2

$243(80.2 \%)$

$93(30.7 \%)$

$28(9.2 \%)$

16 (5.3\%)

$96(31.7 \%)$

$3(1.0 \%)$

$21(6.9 \%)$

$23(7.6 \%)$

$13(4.3 \%)$

$41(13.5 \%)$

49 (16.2\%)

$13(4.3 \%)$

$100 \%$

134 (44.2\%)

$92.3 \%$

177 (63.2\%)

$24(23.1 \%)$

$100 \%$

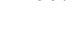

$100 \%$
$100 \%$

$100 \%$
$100 \%$

$\begin{array}{cccc}183(60.3 \%) & 149(63.7 \%) & 34(49.2 \%) & <0.01 \\ 88(43.3 \%) & 63(26.9 \%) & 25(36.2 \%) & 0.01 \\ 19(6.8 \%) & 18(7.7 \%) & 1,0(1.4 \%) & 0.01 \\ 18(5.9 \%) & 12(5.1 \%) & 6(8.7 \%) & 0.15 \\ 120(39.6 \%) & 99(42.3 \%) & 21(30.4 \%) & 0.01 \\ 163(53.8 \%) & 111(47.4 \%) & 52(75.3 \%) & <0.01 \\ 168(55.4 \%) & 99(42.3 \%) & 69(100 \%) & <0.01 \\ 61(20.1 \%) & 0 & 61(88.4 \%) & \\ 30(9.9 \%) & 0 & 30(43.4 \%) & \end{array}$

Group 2

n. 69

n. 234

$103(44.0 \%)$

$50(72.4 \%) \quad<0.01$

49 (21.0\%)

$22(31.9 \%) \quad 0.04$

$36(15.4 \%)$

8 (11.6\%)

0.65

$19(8.1 \%)$

$7(11.1 \%)$

0.67

$24(10.3 \%)$

2 (2.9\%)

0.16

$21(9 \%)$

$5(7.2 \%)$

0.93

$0(0 \%)$

3 (4.3\%)

0.02

$28(12 \%)$

8 (11.6\%)

0.90

$12(5.1 \%)$

$3(4.3 \%)$

0.93

$40(17.1 \%)$

11 (16.0\%)

0.87

119 (50.8\%)

35 (50.7\%)

0.80

87 (37.2\%)

14 (20.3\%)

0.03

71 (30.3\%)

25 (36.2\%)

0.47

187 (79.9\%)

56 (83.2\%)

0.73

59 (25.2\%)

34 (49.3\%)

$<0.01$

24 (10.3\%)

4 (5.8\%)

0.54

$10(4.3 \%)$

$6(8.7 \%)$

0.21

69 (29.5\%)

27 (39.1\%)

0.20

3 (4.3\%)

0.02

17 (7.3\%)

4 (5.8\%)

0.81

16 (6.8\%)

7 (10.1\%)

0.55

10 (4.3\%)

3 (4.3\%)

1

31 (13.2\%)

$10(14.5 \%)$

0.69

40 (17.1\%)

9 (13.0\%)

0.52

12 (5.1\%)

1 (1.4\%)

0.33

97 (41.4\%)

37 (53.6\%)

0.12

$140(60.1 \%)$

37 (78.7\%)

0.15

16 (17.2\%)

$8(72.7 \%)$

0.01

$100 \%$


Table 2 Clinical, radiological characteristics, treatments and follow-up swabs of Group 1 and Group 2 COVID-19 patients (Continued)

\begin{tabular}{|c|c|c|c|c|c|}
\hline & Available & $\begin{array}{l}\text { All patients } \\
\text { n. } 303\end{array}$ & $\begin{array}{c}\text { Group } 1 \\
\text { n. } 234\end{array}$ & $\begin{array}{c}\text { Group } 2 \\
\text { n. } 69\end{array}$ & $P$ \\
\hline Negative follow up swab for SARS-CoV-2 ${ }^{b}$ & $93.7 \%$ & $236(83.1 \%)$ & $182(80.1 \%)$ & $54(91.5 \%)$ & 0.70 \\
\hline Days from hospitalization to negativization & $93.7 \%$ & $22(14-39)$ & $20(13.9-32)$ & $35(20-57)$ & $<0.01$ \\
\hline
\end{tabular}

Data presented as median (IQR) or as percentage. In the second column data availability is also shown

ICU Intensive Care Unit, SICU Sub-intensive Care Unit, IQR Interquartile Range, BMI Body Mass Index, Sat02 Oxygen Saturation, iv intravenous, NIV Non-Invasive Ventilation, MV Mechanical Ventilation

${ }^{a}$ Nasal cannula, face-mask. ${ }^{\text {b}}$ Two negative throat-swabs obtained in consecutive days

The median age was 62 years and the univariate analysis did not show a significant difference between the two groups of patients. This is possibly due to the narrow distribution of the patients in the age class $50-70$ years $(\mathrm{IQR}=47-74)$, which reflects the specific characteristics of our patients' sample: older patients are more likely to present a critical clinical presentation and therefore to be directly admitted or precociously transferred to the ICU or SICU.

The presence of underlying medical disorders was associated with a greater risk to be transferred to the ICU or SICU. Hypertension and known or newly diagnosed diabetes were significantly more represented in Group 2 . The three transplant patients of our cohort (a lung and two kidney transplants) required to be transferred to the SICU, but they all recovered. These data confirm that more fragile patients have a higher risk for severe disease and often require tertiary care management [22].

Fever is normally used as a primary screening tool to identify COVID-19 patients and was also the most common sign in both groups, consistently with other studies $[13,22]$. All the other symptoms were equally reported among the two groups of patients, with the exception of dry cough that was more common in Group 2. Dry cough is typical of interstitial pneumonia and it may reflect the progressive severe pulmonary involvement of patients admitted to the ICU/SICU.

Overall, $63.2 \%$ of patients had pathological findings at the chest $\mathrm{x}$-ray at admission, with no significant differences between the two groups. The homogeneous distribution of X-ray abnormalities may have been influenced by the exclusion of patients presenting a critical clinical condition from the beginning of the hospitalization. However, Group 2 patients were more likely to develop a pathological chest $\mathrm{X}$ ray during the hospital stay. These findings arouse two main observations. First the need of a close monitoring of patients in the early phase of the hospitalization and the suggestion that repeated radiological examinations are useful in identifying patients at risk for complications [23]. Second, the opportunity to consider a computed tomography (CT) chest scan as a first level diagnostic procedure in the context of COVID-19. To date, the best radiological strategy remains undefined. The use of CT-scan for all patients appears be unreasonable in terms of time, costs and radiation exposure [24]. An alternative option could be the combination of chest-X-ray and ultrasound, that has demonstrated a sensitivity of $75 \%$ (vs. $59 \%$ of chest Xray) in detecting an interstitial lung disease $[25,26]$.

In terms of laboratory findings, Group 2 patients presented at admission a significantly higher WBC with neutrophilia, higher levels of AST and increased values of CRP. To identify possible early signals of clinical worsening and considering that the median time from hospital admission to the ICU/SICU transfer was of 5 days, we analysed the differences of laboratory findings at the 3rd measurement since admission: the differences between Group 1 and 2 identified at the beginning of the hospitalization were confirmed, with the inclusion of ALT and total bilirubin. However, WBC counts and hepatic inflammation markers resulted to be only moderately altered in Group 2. It is important to take into account that both high levels of CRP and the alterations of WBC and hepatic inflammation markers could be partly explained by concomitant bacterial infections and medications. Nevertheless, in our cohort, all the microbiological investigations aimed at the detection of bacterial superinfections resulted negative. Therefore it is reasonable to consider the persistent observation of even slightly abnormal WBC count and hepatic inflammation markers and of high levels of CRP as possible markers of disease severity that should lead physicians to a closer follow-up for the risk of clinical worsening. The association between high concentrations of CRP and COVID19 severe clinical presentations has also been reported in several other studies $[9,10,12,16,22]$.

A significant lower level of lymphocyte T CD4+ count was recorded in Group 2 compared to Group 1. Researchers have recently demonstrated that $\mathrm{T}$ cells can have a role in contrasting the SARS-CoV-2 infection and that the severity of the disease can depend on the strength of $T$ cell responses $[27,28]$. A speculation could be that the partial impaired immune system of Group 2 patients may play a detrimental role in the early phase of the infection, even thought it is still unknown whether $\mathrm{T}$ cells can promote the elimination of the virus or even cause a dangerous immune system overreaction [28].

In our cohort, patients transferred to the ICU/SICU resulted to be less likely to receive $\mathrm{HCQ}$ or $\mathrm{CQ}$ and Remdesivir during the course of hospitalization. On the 
other side, those patients were more often prescribed with Lopinavir/ritonavir, possibly because at the early stage of the outbreak this drug was the only one considered effective, and therefore more often used for severe patients. However, no conclusion can be drawn, and the effective role of these drugs used alone or in combination remains uncertain until the results of large controlled randomized studies will be available.

Our study as some limitations: first, the study cohort does not include COVID-19 patients admitted in general wards other than the Infectious Diseases Unit; a larger sample may have revealed additional elements not readily apparent in our series. Second, the observational design has the potential for incomplete capture or misclassification of baseline characteristics. Third, the follow up was not long enough to collect complete outcome data from all the patients.

However, the prospective collection of data, the monocentric design and the focus on the characteristics of the patients requiring either sub-intensive or intensive care represent added values.

\section{Conclusion}

The proper management of COVID-19 patients requires a multilevel diagnostic approach that should be focused on the early recognition of signs of disease severity and progression. The clinical condition of patients primarily admitted to a general ward with a non-critical disease can worsen within few days, requiring the transfer to intensive or sub-intensive specialized units. The results of our study indicate that the presence of comorbidities, such as hypertension and diabetes, and of dry cough are potentially associated with a negative progression of the disease. In addition, not only laboratory alterations at admission, but also the persistent observation of abnormal laboratory findings should be regarded with a high index of suspicion for clinical worsening. The availability of tertiary level hospital with adequate provision of subintensive and intensive care is a crucial part of the response to the current and possible future outbreaks.

\section{Supplementary Information}

The online version contains supplementary material available at https://doi. org/10.1186/s12879-020-05647-7.

Additional file 1: Table 3. Laboratory findings at $1^{\circ}, 3^{\circ}$ and last

measurement of Group 1 and Group 2 patients.

\footnotetext{
Abbreviations

ALP: Alkaline Phosphatase; ALT: Alanine Aminotransferase; APTT: Activated Partial Thromboplastin Time; AST: Aspartate Aminotransferase; COVID-

19: Coronavirus Diseases 2019; CQ: Chloroquine; CRP: C-Reactive-Protein; GFR: Glomerular Filtration Rate; GGT: Gamma-Glutamyl-Transferase; HCQ: Hydroxychloroquine; ICU: Intensive Care Unit; IDU: Infectious Diseases Unit; IQR: Inter-quartile Range; MV: Mechanical Ventilation; NIV: Non Invasive Ventilation; PT: Prothrombin Time; RT-PCR: Real Time Polymerase Chain
}

Reaction; SARS-CoV-2: Severe Acute Respiratory Syndrome Coronavirus 2; SICU: Sub-Intensive Care Unit

\section{Acknowledgements}

We thank the whole medical and nursing team of the Infectious Diseases Unit and, most of all, the patients.

\section{Authors' contributions}

All authors contributed to the study conception and design. Material preparation, data collection and analysis were performed by EDM, AF1, LS, AF2, MV and FG. MT and GC contributed to the design and implementation of the research and to the critical interpretation of the results. The first draft of the manuscript was written by AMC, LS and EDM and all authors commented on previous versions of the manuscript. All authors read and approved the final manuscript.

\section{Funding}

The authors did not receive additional funding for the work.

\section{Availability of data and materials}

The Author confirms that the data supporting the findings of this study are available within the article. More information that supports the findings of this study is available from the corresponding author, $A M C$, upon reasonable request.

\section{Ethics approval and consent to participate}

Ethical approval for this study was obtained from the Institutional Review Board of the University of Padua. In agreement with Italian national regulation on retrospective observational studies that do not imply experimentation on the patients or deviation from the standard clinical practice, the need for patient's written informed consent was waived. All participants gave instead verbal informed consent to the treating physician with the presence of a witness. The expressed consent was reported on the patient's clinical file together with the agreement of the personal data handling, which is always obtained at the beginning of hospitalization. All analyses were carried out on anonymised data.

\section{Consent for publication}

Not applicable.

\section{Competing interests}

The authors declare that they have no competing interests.

\section{Author details}

'Infectious Diseases Unit, Azienda Ospedale Università di Padova, Via Nicolò Giustiniani 2, 35128 Padova, Italy. ${ }^{2}$ Information Technology System Unit, Azienda Ospedale Università di Padova, Padova, Italy. ${ }^{3}$ Department of Directional Hospital Management, Azienda Ospedale Università di Padova, Padova, Italy.

Received: 2 July 2020 Accepted: 22 November 2020

Published online: 09 December 2020

References

1. WHO. WHO Director-General's opening remarks at the media briefing on COVID-19 2020. https://www.who.int/dg/speeches/detail/who-directorgeneral-s-opening-remarks-at-the-media-briefing-on-covid-19\%2D\%2D-11march-2020. Accessed 10 June 2020.

2. Ministero Della Salute. Novel Coronavirus - Situation report. http://www. salute.gov.it/portale/nuovocoronavirus/ dettaglioContenutiNuovoCoronavirus.jsp?lingua=italiano\&id=5351\&area= nuovoCoronavirus\&menu=vuoto. Accessed 10 June 2020.

3. Saglietto A, D'Ascenzo F, Zoccai GB, De Ferrari GM. COVID-19 in Europe: the Italian lesson. Lancet. 2020;395:1110-1. https://doi.org/10.1016/S01406736(20)30690.

4. Grasselli G, Pesenti A, Cecconi M. Critical care utilization for the COVID-19 outbreak in Lombardy, Italy: early experience and forecast during an emergency response. JAMA. 2020. https://doi.org/10.1001/jama.2020.4031.

5. Verity R, Okell LC, Dorigatti I, et al. Estimates of the severity of coronavirus disease 2019: a model based analysis. Lancet Infect Dis. 2020;0. https://doi. org/10.1016/S1473-3099(20)30243-7. 
6. World Health Organization. Clinical management of COVID-19: interim guidance. 2020. https://www.who.int/publications//item/clinicalmanagement-of-covid-19. Accessed 2 June 2020.

7. Rhodes A, Ferdinande P, Flaatten H, Guidet B, Metnitz PG, Moreno RP. The variability of critical care bed numbers in Europe. Intensive Care Med. 2012; 38(10):1647-53. https://doi.org/10.1007/s00134-012-2627-8.

8. Chen T, Wu D, Chen H, et al. Clinical characteristics of 113 deceased patients with coronavirus disease 2019: retrospective study. BMJ. 2020;368. https://doi.org/10.1136/bmj.m1091.

9. Guan WJ, Ni ZY, Hu Y, et al. Clinical Characteristics of Coronavirus Disease 2019 in China. N Engl J Med. 2020;382:1708-20. https://doi.org/10.1056/ NEJMoa2002032

10. Bhargava A, Fukushima EA, Levine $M$, et al. Predictors for severe COVID-19 infection. Clin Infect Dis. 2020. https://doi.org/10.1093/cid/ciaa674.

11. Buckner FS, McCulloch DJ, Atluri V, et al. Clinical Features and Outcomes of 105 Hospitalized patients with COVID-19 in Seattle, Washington [published online ahead of print, 2020 May 22]. Clin Infect Dis. 2020;ciaa632. https://doi.org/10.1093/cid/ciaa632.

12. Petrilli CM, Jones SA, Yang J, et al. Factors associated with hospita admission and critical illness among 5279 people with coronavirus disease 2019 in New York City: prospective cohort study. BMJ. 2020;369:m1966. Published 2020 May 22. https://doi.org/10.1136/bmj.m1966.

13. Docherty AB, Harrison EM, Green CA, et al. Features of 20133 UK patients in hospital with covid-19 using the ISARIC WHO clinical characterisation protocol: prospective observational cohort study. BMJ. 2020;369. https://doi.org/10.1136/bmj.m1985.

14. Bhatraju PK, Ghassemieh BJ, Nichols M, et al. Covid-19 in critically ill patients in the Seattle region: case series. N Engl J Med. 2020. https://doi.org/10. 1056/NEJMoa2004500.

15. Grasselli G, Zangrillo A, Zanella A, et al. Baseline characteristics and outcomes of 1591 patients infected with SARS-CoV-2 admitted to ICUs of the Lombardy region, Italy JAMA Published online April 06, 2020. https:// doi.org/10.1001/jama.2020.5394.

16. Hou W, Zhang W, Jin R, Liang L, Xu B, Hu Z. Risk factors for disease progression in hospitalized patients with COVID-19: a retrospective cohort study. Infect Dis (Lond). 2020;52:498-505. https://doi.org/10.1080/23744235. 2020.1759817.

17. DaweiWang MD, Bo Hu MD, Chang Hu MD, Fangfang Zhu MD, Xing Liu MD, Jing Zhang MD, BinbinWang MD, et al. Clinical characteristics of 138 hospitalized patients with 2019 novel coronavirus-infected pneumonia in Wuhan, China. JAMA. 2020. https://doi.org/10.1001/jama.2020.1585.

18. Mehta S, Hill NS. Non-invasive Ventilation. Am J RespirCrit Care Med. 2001; 163:540-77. https://doi.org/10.1164/ajrccm.163.2.9906116.

19. Lavezzo E, Franchin E, Ciavarella C, et al. Suppression of a SARS-CoV-2 outbreak in the Italian municipality of Vo'. Nature. 2020. https://doi.org/10. 1038/s41586-020-2488-1.

20. SIMIT (Societàltaliana di Malattielnfettive e Tropicali) SEZIONE REGIONE LOMBARDIA. Vademecum per la curadellepersone con malattia da COVID-19 Edizione 2.0, 2020. https:/www.sicp.it/aggiornamento/linee-guida-bpprocedures/2020/04/pandemia-covid-19-sars-cov-2-26. Accessed 1 June 2020

21. Jaillon $S$, Berthenet $K$, Garlanda C. Sexual dimorphism in innate immunity. Clin Rev Allergy Immunol. 2019;56:308-21. https://doi.org/10.1007/s12016017-8648-x.

22. Chen $N$, Zhou M, Dong $X$, et al. Epidemiological and clinical characteristics of 99 cases of 2019 novel coronavirus pneumonia in Wuhan, China: a descriptive study. Lancet. 2020;395:507-13. https://doi.org/10.1016/S01406736(20)30211-7.

23. Yang $X, Y u Y, X u J$, et al. Clinical course and outcomes of critically ill patients with SARS-CoV-2 pneumonia in Wuhan, China: a single-centered, retrospective, observational study. Lancet Respir Med. 2020;8:475-81. https://doi.org/10.1016/S2213-2600(20)30079-5.

24. Pascarella G, Strumia A, Chiara Piliego C, et al. COVID-19 diagnosis and management: a comprehensive review. J Intern Med. 2020. https://doi.org/ 10.1111/joim.13091.

25. Bernheim A, Mei $X$, Huang $M$, et al. Chest $C T$ findings in coronavirus disease-19 (COVID- 19): relationship to duration of infection. Radiology. 2020;200463. https://doi.org/10.1148/radiol.2020200463.

26. Yi Huang S, Liu Y, Zhang Y, ChuyunZheng Y, Zheng CZ, Min W, Ming Y, Mingjun $\mathrm{H}$. A preliminary study on the ultrasonic manifestations of peripulmonary lesions of non- critical novel coronavirus pneumonia (COVID19). Res Square. https://doi.org/10.21203/rs.2.24369/v1.
27. Chen G, Wu D, Guo W, et al. Clinical and immunological features of severe and moderate coronavirus disease 2019. J Clin Invest. 2020;130(5):2620-9. https://doi.org/10.1172/JCl137244.

28. Grifoni A, Weiskopf D, Ramirez SI, et al. Targets of T cell responses to SARSCoV-2 coronavirus in humans with COVID-19 disease and unexposed individuals. Cell. 2020; S0092-8674(20)30610-3. https://doi.org/10.1016/j.cell. 2020.05.015.

\section{Publisher's Note}

Springer Nature remains neutral with regard to jurisdictional claims in published maps and institutional affiliations.

\section{Ready to submit your research? Choose BMC and benefit from:}

- fast, convenient online submission

- thorough peer review by experienced researchers in your field

- rapid publication on acceptance

- support for research data, including large and complex data types

- gold Open Access which fosters wider collaboration and increased citations

- maximum visibility for your research: over $100 \mathrm{M}$ website views per year

At BMC, research is always in progress.

Learn more biomedcentral.com/submissions 